\title{
Quinoline-based antimalarial drugs: a novel class of autophagy inhibitors
}

\author{
Encouse B. Golden, MD, PhD, ${ }^{1}$ Hee-Yeon Cho, PhD, ${ }^{2}$ Florence M. Hofman, PhD, ${ }^{3}$ \\ Stan G. Louie, PhD, ${ }^{4}$ Axel H. Schönthal, PhD, ${ }^{5}$ and Thomas C. Chen, MD, PhD ${ }^{2,3}$ \\ 1Department of Radiation Oncology, New York University School of Medicine, New York, New York; Departments of \\ ${ }^{2}$ Neurosurgery, ${ }^{3}$ Pathology, and ${ }^{5}$ Molecular Microbiology and Immunology, Keck School of Medicine of USC; and ${ }^{4}$ Department of \\ Pharmaceutical Sciences, School of Pharmacy, University of Southern California, Los Angeles, California
}

OBJECT Chloroquine (CQ) is a quinoline-based drug widely used for the prevention and treatment of malaria. More recent studies have provided evidence that this drug may also harbor antitumor properties, whereby CQ possesses the ability to accumulate in lysosomes and blocks the cellular process of autophagy. Therefore, the authors of this study set out to investigate whether $\mathrm{CQ}$ analogs, in particular clinically established antimalaria drugs, would also be able to exert antitumor properties, with a specific focus on glioma cells.

METHODS Toward this goal, the authors treated different glioma cell lines with quinine (QN), quinacrine (QNX), mefloquine (MFQ), and hydroxychloroquine (HCQ) and investigated endoplasmic reticulum (ER) stress-induced cell death, autophagy, and cell death.

RESULTS All agents blocked cellular autophagy and exerted cytotoxic effects on drug-sensitive and drug-resistant glioma cells with varying degrees of potency (QNX $>M F Q>H C Q>C Q>Q N)$. Furthermore, all quinoline-based drugs killed glioma cells that were highly resistant to temozolomide (TMZ), the current standard of care for patients with glioma. The cytotoxic mechanism involved the induction of apoptosis and ER stress, as indicated by poly(ADP-ribose) polymerase (PARP) cleavage and CHOP/GADD153. The induction of ER stress and resulting apoptosis could be confirmed in the in vivo setting, in which tumor tissues from animals treated with quinoline-based drugs showed increased expression of CHOP/GADD153, along with elevated TUNEL staining, a measure of apoptosis.

CONCLUSIONS Thus, the antimalarial compounds investigated in this study hold promise as a novel class of autophagy inhibitors for the treatment of newly diagnosed TMZ-sensitive and recurrent TMZ-resistant gliomas.

http://thejns.org/doi/abs/10.3171/2014.12.FOCUS14748

KEY WORDS glioma; chloroquine; antimalaria compound; autophagy; endoplasmic reticulum stress

$\mathrm{G}$ LIOBLASTOMA multiforme (GBM) is a malignant brain tumor associated with a poor prognosis. Temozolomide (TMZ) is the conventional chemotherapy for patients with GBM; however, patients usually develop TMZ resistance and succumb to death. Chloroquine (CQ) in combination with conventional GBM therapy recently demonstrated the ability to prolong median survival from 11.4 to 25 months in patients with GBM. ${ }^{6}$ The mechanism by which CQ enhances conventional GBM therapy is not well understood.
Quinoline-based antimalarial (QBA) compounds are weak bases that accumulate in the acidic food vacuoles (AFVs) of Plasmodium falciparum parasites. ${ }^{37}$ The chemical structures of several QBAs are displayed in Fig. 1. Chloroquine, the prototypical antimalarial compound, interferes with ferriprotoporphyrin IX detoxification in the AFVs of P. falciparum, thereby causing parasite death. ${ }^{1,2,19,20,26}$ In addition to AFVs, CQ has been shown to accumulate in the acidic lysosomes of tumor cells, thereby disrupting autophagy. ${ }^{1,9,15}$

ABBREVIATIONS AFV = acidic food vacuole; $\mathrm{CFA}=$ colony formation assay; $\mathrm{CQ}=$ chloroquine; $\mathrm{ER}=$ endoplasmic reticulum; $\mathrm{GBM}=\mathrm{glioblastoma}$ multiforme; $\mathrm{HCQ}=$ hydroxychloroquine; $I_{50}=$ half maximal inhibitory concentration; $I H C=$ immunohistochemistry; $M F Q=$ mefloquine; $P A R P=$ poly $(A D P-$ ribose $)$ polymerase; $Q B A=$ quinolinebased antimalarial; $\mathrm{QN}=$ quinine; $\mathrm{QNX}=$ quinacrine; $\mathrm{TMZ}=$ temozolomide; U251-TMZR cells = TMZ-resistant U251 cells.

SUBMITTED October 31, 2014. ACCEPTED December 12, 2014

INCLUDE WHEN CITING DOI: 10.3171/2014.12.FOCUS14748.

DISCLOSURE The authors report no conflict of interest concerning the materials or methods used in this study or the findings specified in this paper. 
A

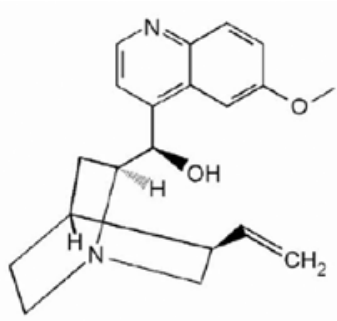

\section{Quinine} (QN)

C<smiles>CCN(CCO)CCCC(C)Nc1ccnc2cc(Cl)ccc12</smiles>

Hydroxychloroquine

E<smiles>O[C@H](c1cc(C(F)(F)F)nc2c(C(F)(F)F)cccc12)[C@H]1CCCCN1</smiles>

\section{Mefloquine} (MFQ)
B<smiles>CCN(CC)CCCC(C)Nc1ccnc2cc(Cl)ccc12</smiles>

Chloroquine

D<smiles>CCN(CC)CCCC(C)Nc1c2cc(Cl)ccc2nc2ccc(OC)cc12</smiles>

Quinacrine (QNX)

FIG. 1. Chemical structures of QBAs: QN (A), CQ (B), HCQ (C), QNX (D), and MFQ (E).

The AFVs of the malaria-causing parasite, $P$. falciparum, catabolize erythrocyte-derived hemoglobin to provide bioenergetic support for parasite growth and replication. ${ }^{18,26}$ Similarly, tumor cell lysosomes degrade damaged organelles or bulk proteins for amino acid and energy recycling via the autophagy pathway. ${ }^{8}$

Quinoline-based antimalarial compounds, such as CQ, hydroxychloroquine (HCQ), quinine (QN), mefloquine (MFQ), and quinacrine (QNX), are mainstay therapies that effectively kill P. falciparum parasites. Chloroquine, a commonly prescribed QBA that harbors weakly basic properties, traverses erythrocyte and $P$. falciparum cellular membranes down a $\mathrm{pH}$ gradient to accumulate inside AFVs and disrupt heme detoxification, thereby causing parasite death. Hydroxychloroquine has antimalarial activity similar to that of CQ; however, since HCQ has a lower associated risk of ocular toxicity, it is clinically preferred over CQ when high doses are necessary for the treatment of malaria. ${ }^{17,27}$ Quinine was the initial QBA isolated from tree bark powder of the Cinchona species. ${ }^{40} \mathrm{~A}$ 3- to 7-day regimen of $\mathrm{QN}$ has rates of efficacy ranging

from $54 \%$ to $95 \%$ against uncomplicated malaria caused by $P$. falciparum throughout different regions in the world. ${ }^{4}$ Mefloquine and QNX have been shown to be efficacious in CQ-resistant malaria. ${ }^{7,11,28,33,41}$ Quinine, MFQ, and QNX have demonstrated the ability to penetrate the blood-brain barrier; therefore, QN and MFQ are effectively used to treat malaria-causing parasites that have entered the central nervous system. . $^{3,5,21,22,32}$

In addition to targeting the AFVs of $P$. falciparum, CQ has recently received attention for its antitumor effects, where it was shown to accumulate in the lysosomes of tumor cells, blocking autophagy and preventing the degradation of autophagosomal content. Moreover, we have recently examined the use of MFQ in breast cancer. ${ }^{35}$

Since QBAs possess the unique ability to accumulate in the AFVs of P. falciparum in various clinical conditions, we set out to determine whether these compounds have antitumor properties similar to those of CQ, including the ability to accumulate in lysosomes and block autophagy. Our data here showed that QBAs induced higher levels of CHOP/GADD-153 (an endoplasmic reticulum [ER] stress proapoptotic protein) as well as apoptosis in vitro and in vivo in U87MG subcutaneous implants. These data indicate that QBA compounds are a novel class of clinically useful autophagy inhibitors that accumulate in lysosomes and block autophagosome degradation, thereby activating ER stress and induced apoptosis in tumor cells.

\section{Methods \\ Reagents}

Chloroquine, MFQ, QNX, QN, and HCQ were all purchased from Sigma (St. Louis, MO). The CQ, HCQ, QNX, and QN were dissolved in ultrapure water at $100-\mu \mathrm{M}$ concentrations. Mefloquine was dissolved in dimethyl sulfoxide (DMSO) at $100-\mu \mathrm{M}$ concentrations. All compounds were stored at $-20^{\circ} \mathrm{C}$.

\section{Cell Lines and Culturing}

Human glioma cell lines (LN229 and U251) were kindly supplied by Dr. Frank Furnari. The U87 glioma cells were purchased from American Type Culture Collection. Glioma cells were propagated in DMEM (Cellgro) supplemented with $10 \%$ fetal bovine serum. All cell lines were grown in $100 \mathrm{U} / \mathrm{ml}$ penicillin and $0.1 \mathrm{mg} / \mathrm{ml}$ streptomycin in a humidified incubator at $37^{\circ} \mathrm{C}, 5 \% \mathrm{CO}_{2}$ atmosphere.

\section{In Vivo Subcutaneous Glioma Rodent Model}

All animal protocols were approved by the University of Southern California Department of Animal Resources, and animals were maintained according to approved guidelines. Tumor cells $\left(2 \times 10^{5}\right)$ were injected subcutaneously into the right flank of athymic nude mice. After tumors were palpable, animals were treated with vehicle or drugs for 24 or 48 hours. Subsequently, the animals were euthanized, and tumor tissues were harvested and prepared for immunohistochemistry (IHC) staining, as described below.

\section{MTT Assay}

The MTT assay, a measure of cell viability, was per- 
formed in 96-well plates using $3.0 \times 10^{3}$ cells per well. ${ }^{25}$ In individual experiments, each treatment condition was set up in quadruplicate, and each experiment was repeated $1-5$ times independently.

\section{Colony Formation Assay}

Cells were seeded into 6-well plates at 200 cells per well. After complete cell adherence, the cells were exposed to drug treatment for 48 hours (in triplicate). Thereafter, the drug was removed, fresh growth medium was added, and the cells were kept undisturbed in culture for 12-14 days. Subsequently, colonies of proliferating cells were visualized by staining with $1 \%$ methylene blue (in methanol) for 4 hours and then were counted.

\section{Light Microscopy}

Digital images of cells in culture were captured with a SPOT camera, model 1.4.0, operated with Spot Advanced Software version 4.0.8 (Diagnostic Instruments) and saved in a 12-bit grayscale format with $1600 \times 1200$ active pixels.

\section{Immunofluorescent and IHC Staining}

Tumor tissues were harvested when the mice were euthanized, snap frozen, and cut into 8- $\mu \mathrm{m}$ sections. Immunofluorescent staining was performed with the use of rabbit primary antibodies targeting LC3B purchased from Cell Signaling Technologies. Texas red-conjugated goat antirabbit IgG was used as a secondary antibody and fluorescent probe (Vector Laboratories). For IHC, after the primary antibody, the biotinylated secondary antibody was applied (Vector Laboratories). After incubations with primary and secondary antibodies were completed, the slides were prepared using blue-fluorescent DAPI dye mounting medium (Vector Laboratories) as a nuclear counterstain or hematoxylin for IHC. All antibodies and nuclear stains were used in accordance with the manufacturer's recommendations. Fluorescent staining was visualized using the Leica DM LB2 universal system microscope (Leica Microsystems Inc.) at a 540-nm excitation wavelength.

\section{Quinacrine Intracellular Fluorescence}

Digital images of QNX intracellular fluorescence in cultured cells treated with or without QNX were taken using the Leica DM LB2 universal system microscope; the excitation wavelength was $470 \mathrm{~nm}$.

\section{Immunoblots and Antibodies}

Preparation of the cell lysates and determination of the protein concentration were performed as previously described.$^{25}$ Lysate $(50 \mu \mathrm{g})$ from each sample was run in parallel. Primary antibodies were purchased from Cell Signaling Technologies or Santa Cruz Biotechnology Inc. and were used according to the manufacturers' instructions. Blocking buffer and fluorescent-conjugated secondary antibodies were purchased from LI-COR Biosciences and used according to protocols supplied by the manufacturer. The membranes were scanned and analyzed with the Odyssey Infrared Imaging System (LI-COR Biosciences) according to the manufacturer's instructions. All immunoblots were repeated at least once.

\section{Proteasome Activity Assay}

Proteasome activity was determined via the direct addition of drugs to purified proteasomes. For purified proteasome activity, increasing concentrations of CQ were added to proteasome assay buffer $(25 \mathrm{mM}$ HEPES, $\mathrm{pH}$ 7.5; 0.5 mM EDTA; $0.05 \%$ NP-40; and $0.001 \%$ sodium dodecyl sulfate $[\mathrm{w} / \mathrm{v}])$, incubated for 30 minutes at room temperature, and then added to $0.2 \mu \mathrm{g}$ of isolated $20 \mathrm{~S}$ proteasomes (Sigma-Aldrich). After 5 minutes of incubation at room temperature, proteasome activity was measured using a $20 \mathrm{~S}$ proteasome activity assay kit. The emitted fluorescence of cleaved proteasome substrate (LLVY-AMC) was detected with a 380/460-nm filter. Cleaved LLVYAMC is indicative of chymotryptic activity.

\section{Results}

\section{Cytotoxicity of QBAs for Glioma Cells}

To investigate the cytotoxic effects of CQ on glioma cells, U251 (p53 mutant, PTEN mutant), LN229 (p53 mutant, PTEN wild type), and U87 ( $p 53$ wild type, PTEN mutant) glioma cell lines were treated with increasing concentrations of CQ for 48 hours. ${ }^{36,42}$ Cytotoxicity was evaluated using the MTT assay; the half maximal inhibitory concentration $\left(\mathrm{IC}_{50}\right.$; that is, the drug dose that causes $50 \%$ cell death) of these glioma cell lines was approximately $30 \mu \mathrm{M}$ (Fig. 2A). Cell death was independent of p53 and PTEN status.

To determine whether other QBA compounds could mimic the cytotoxic effects of CQ in glioma cells, we used MFQ and QNX. Results showed that these drugs effectively killed U251 cells at much lower concentrations than CQ (Fig. 2B). The viability value for untreated cells over 48 hours was set at $100 \%$, based on previous experience showing that there is minimal cell death in medium alone. Values were calculated as the ratio of cell viability of drug-treated cells relative to that of untreated cultures. Quinoline was less effective in killing glioma cells in vitro than CQ. The $\mathrm{IC}_{50}$ for MFQ, QNX, and QN in U251 cells was 10,5 , and $>100 \mu \mathrm{M}$, respectively. Similar results were seen in LN229 and U87 cells (data not shown).

To determine whether the cytotoxic effects of CQ and its quinoline-based analogs could overcome TMZ resistance in glioma cells, we treated U251 and TMZ-resistant U251 (U251-TMZR) cells with CQ, QNX, MFQ, and QN for 48 hours in parallel MTT assays (Fig. 2C). We found that U251 and U251-TMZ ${ }^{\mathrm{R}}$ cells respond to the QBA compounds at similar doses. The U251 cells' $\mathrm{IC}_{50}$ for CQ, MFQ, and QNX was 40,15 , and $8 \mu \mathrm{M}$, respectively; the $\mathrm{U}^{251-T M Z}{ }^{\mathrm{R}}$ cells' $\mathrm{IC}_{50}$ for CQ, MFQ, and QNX was 40, 12 , and $10 \mu \mathrm{M}$, respectively. Similar results were obtained for LN229/LN229-TMZ ${ }^{\mathrm{R}}$ and U87/U87-TMZ ${ }^{\mathrm{R}}$ tumor cell pairs (data not shown). The LN229 cells' mechanism for resistance has been established to be DNA mismatch repair deficiency. Resistant U251 cells are associated with the overexpression of base excision repair proteins. ${ }^{14}$ The $\mathrm{TMZ}^{\mathrm{R}}$ cells were also tested using the long-term colonyformation assay (CFA; Fig. 2D). Data in the present study showed that in the CFA, $100 \mu \mathrm{M}$ of TMZ reduced the percent of colonies formed in U251 cells by approximately 95\%; in contrast, $100 \mu \mathrm{M}$ of $\mathrm{TMZ}$ reduced the percent 

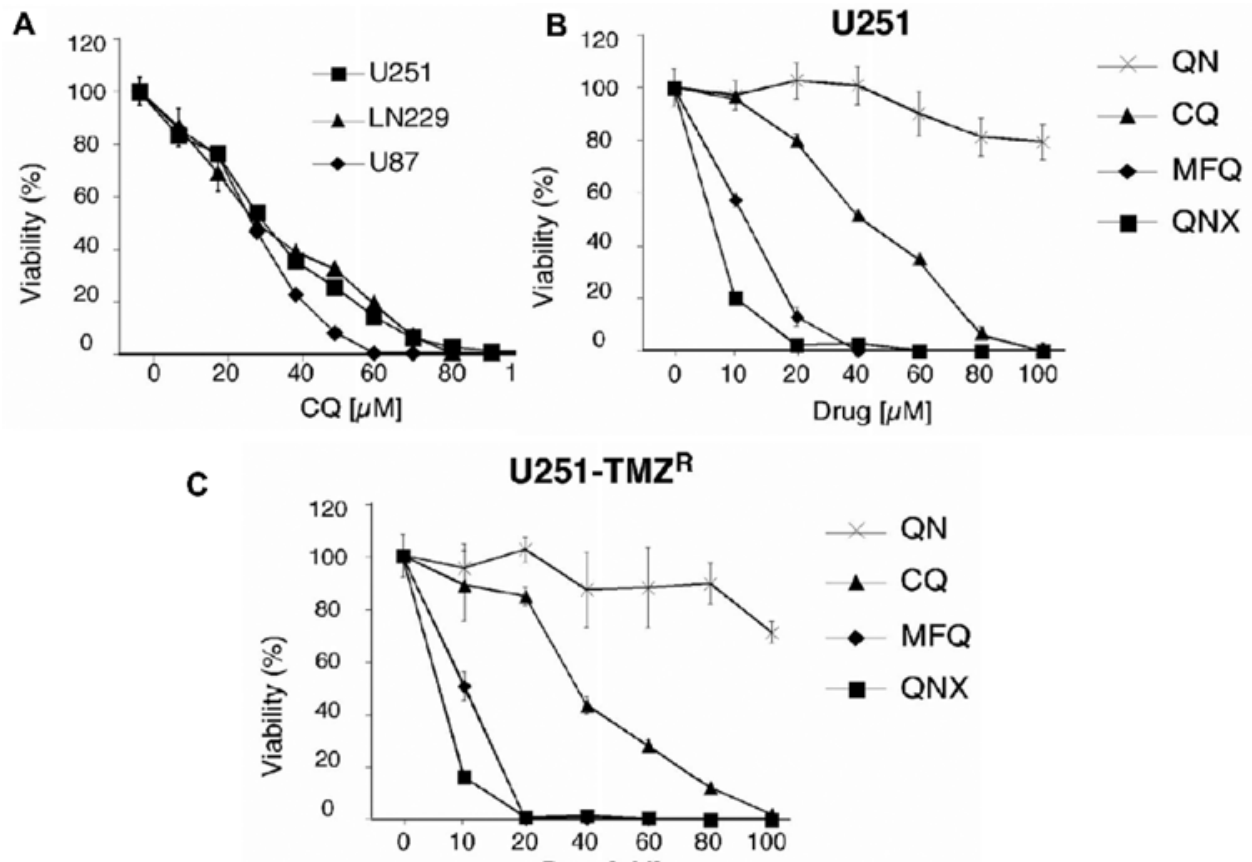

U251-TMZR
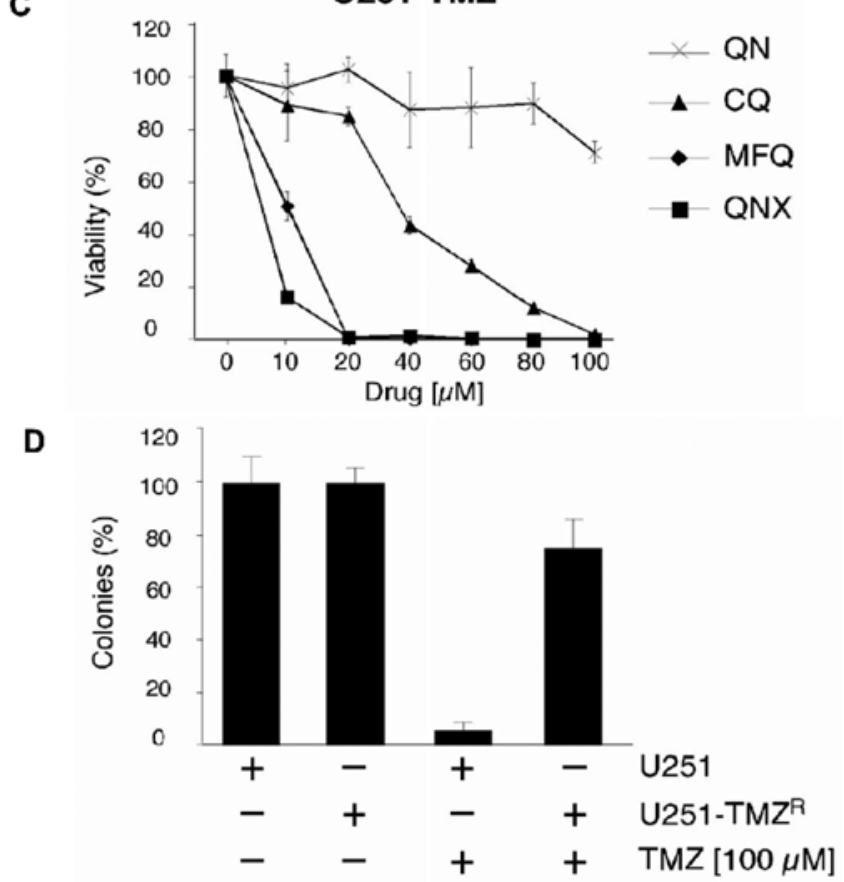

FIG. 2. Quinoline-based antimalarial compounds are cytotoxic to glioma cells. In an MTT assay, U251, LN229, and U87 glioma cell lines were treated with increasing concentrations of CQ (A) for 48 hours. In an MTT assay, the U251 (B) and U251-TMZR (C) glioma cell lines were treated with increasing concentrations of QN, CQ, MFQ, and QNX for 48 hours. The number of viable cells from the untreated controls was set at $100 \%$. Whiskers represent standard deviation. Both U251 and U251-TMZR cells (200 cells/ well) were seeded in 6-well plates and allowed to attach to the plates overnight. Thereafter, the cells were either untreated or treated with $100 \mu \mathrm{M}$ TMZ. The drug was removed, fresh growth medium was added, and the cells were kept in culture undisturbed for 12-14 days, when the surviving cells spawned a colony of proliferating cells (D). Colonies were visualized by staining with $1 \%$ methylene blue (in methanol) for 4 hours and then were counted. Bars represent the mean percentage of surviving cells from triplicate experiments. The number of colonies obtained from the untreated controls was set at $100 \%$.

of colonies formed in $\mathrm{U} 251-\mathrm{TMZ}^{\mathrm{R}}$ by only approximately $25 \%$. We confirmed TMZ resistance in the LN229-TMZ ${ }^{\mathrm{R}}$ and U87-TMZ ${ }^{\mathrm{R}}$ cells with CFA and long-term MTT assays (data not shown). These results showed that QBAs are cytotoxic to TMZ-sensitive and TMZ-resistant glioma cells, regardless of the $p 53$ and PTEN status or the mechanism of resistance to TMZ.

\section{Autophagosome Accumulation and Induction of ER Stress-Induced Apoptosis by Chloroquine}

Chloroquine blocks autophagy in tumor cells; however, the mechanism of this cell death pathway is not well understood. To investigate CQ's mechanism of action, we treated U251 cells with CQ $(50 \mu \mathrm{M})$; after 48 hours, the CQ-treated U251 cells (Fig. 3A, left 2 columns) displayed an accumulation of cytoplasmic vacuoles, as compared with untreated controls. To identify the CQ-induced vacuolar accumulation, the CQ-treated cells were stained with LC3B, an autophagosome marker (Fig. 3A, right 4 columns). Staining revealed an increase in the intensity and amount of LC3B accumulation in the CQ-treated U251 cells.

To confirm the presence of autophagosome accumulation in CQ-treated glioma cells, conversion of the cytoplasmic form of LC3B (LC3B-I) to the autophagosomal membrane-bound form (LC3B-II) was evaluated using LN229 glioma cells (Fig. 3B). Chloroquine increased the conversion of LC3B-I to LC3B-II. Furthermore, CQ treatment caused an increase in the induction of $\mathrm{CHOP} /$ GADD-153 (an ER stress proapoptotic protein) expression 


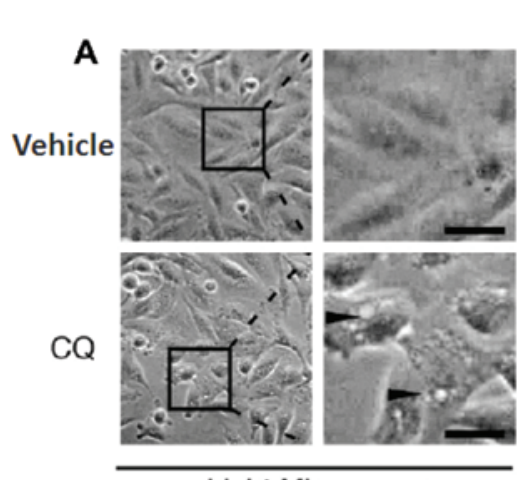

Light Microscopy

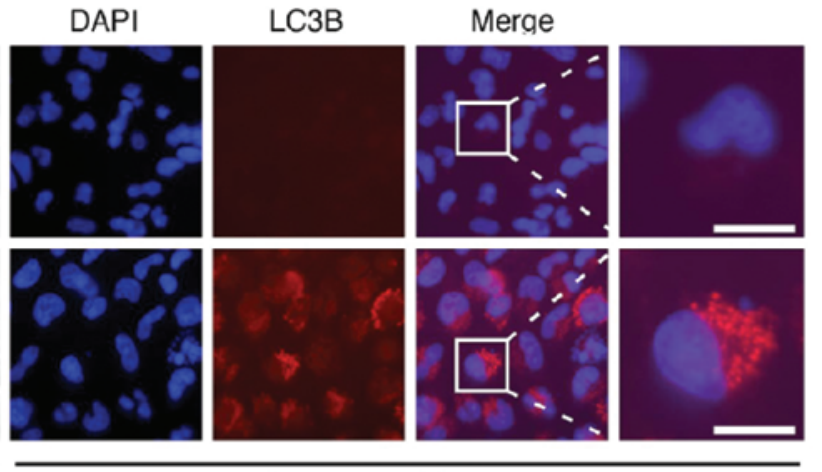

Fluorescent Microscopy
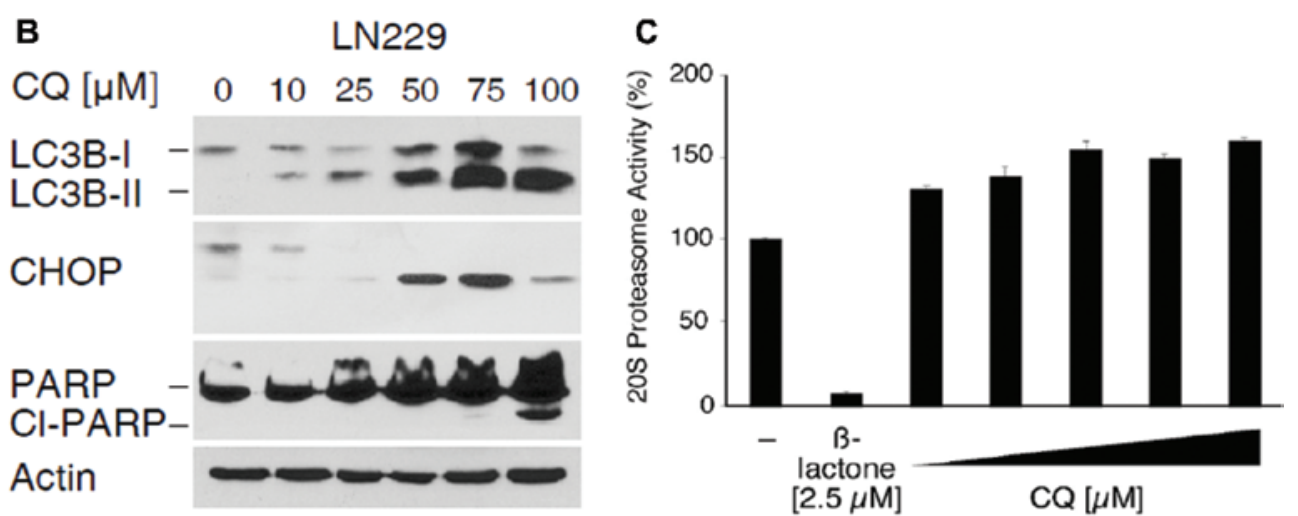

FIG. 3. Chloroquine and HCQ block autophagy and induce apoptosis. Light microscopy images (A, left 2 columns) of U251 cells treated with $50 \mu \mathrm{M}$ of $\mathrm{CQ}$ for 48 hours. Black arrows indicate intracellular vacuoles. Black squares indicate the areas magnified in the right adjacent images. Fluorescent microscopy images (A, right 4 columns) of U251 cells treated with $50 \mu \mathrm{M}$ of CQ for 48 hours and stained with the nuclear stain DAPI (blue) and an immunofluorescent stain for LC3B (red). Red punctate staining indicates the presence of autophagosomes. White squares encompass the area magnified in the right adjacent image. Bar $=10 \mu \mathrm{m}$. LN229 glioma cells were treated with increasing concentrations of CQ or HCQ, respectively, for 24 hours, and relative amounts of LC3B-I, LC3B-II, CHOP/GADD-153, and PARP were analyzed via Western blot (B and C). $\beta$-actin (actin) was used as a loading control. Purified proteasome was treated with increasing concentrations of $C Q$ and analyzed via $20 S$ proteasome activity assay, in which untreated proteasomes are presented as having 100\% 20S proteasome activity. Cl-PARP = PARP cleavage.

and cleavage of the DNA repair enzyme poly(ADP-ribose) polymerase (PARP; PARP cleavage is an apoptosis marker; Fig. 3B).

To determine whether CQ can inhibit isolated 20S proteasomes at relevant cytoplasmic concentrations, isolated proteasomes were treated with increasing concentrations of CQ from 0 to $40 \mu \mathrm{M}$. Data indicated that CQ can stimulate proteasome activity approximately $60 \%$ (Fig. 3C); however, treatment with lactacystin $(2.5 \mu \mathrm{M})$, a proteasome inhibitor, showed proteasome activity was reduced to approximately $90 \%$. Thus, CQ induces apoptosis by blocking the degradation of autophagosomes, but not as a result of proteasome inhibition.

\section{Blocking Autophagy and Inducing Apoptosis in Glioma Cells}

Based on information that CQ induces apoptosis by blocking autophagy, we analyzed whether the other QBA compounds can function in a similar manner. U251 cells were treated with increasing concentrations of $\mathrm{CQ}, \mathrm{QN}$, HCQ, QNX, and MFQ and were tested for the conversion of LC3B-I to LC3B-II, induction of CHOP/GADD153, and cleavage of PARP. The U251 cells treated with increasing concentrations of QBA compounds were able to mimic the cytotoxic effects of CQ (Fig. 4).

Each compound increased the conversion of LC3B-I to LC3B-II, the levels of polyubiquitinated proteins, the induction of CHOP/GADD-153, and the cleavage of PARP, in direct correlation with the drug's ability to reduce viability. Quinacrine, the most potent QBA, not only blocked autophagy, but at $20 \mu \mathrm{M}$ it completely disrupted U251 cellular functioning, which was evidenced by a high level of PARP cleavage, as well as the absence of CHOP/GADD153 and LC3B expression. These data showed that QBAs represent a novel class of compounds with the ability to inhibit autophagy and induce apoptosis in tumor cells.

\section{Accumulation of QNX in Acidic Vacuoles and Cell Death}

Quinacrine is the most potent QBA that we tested. It caused autophagosome accumulation, CHOP/GADD-153 induction, and PARP cleavage in a manner similar to CQ. However, it is unclear whether QNX initiates this activity in the same way as CQ. Since QNX fluoresces, it is possible to determine whether it accumulates within the acidic vacuoles, for example, lysosomes and late endosomes. To accomplish this, U251 cells were treated with QNX (10 

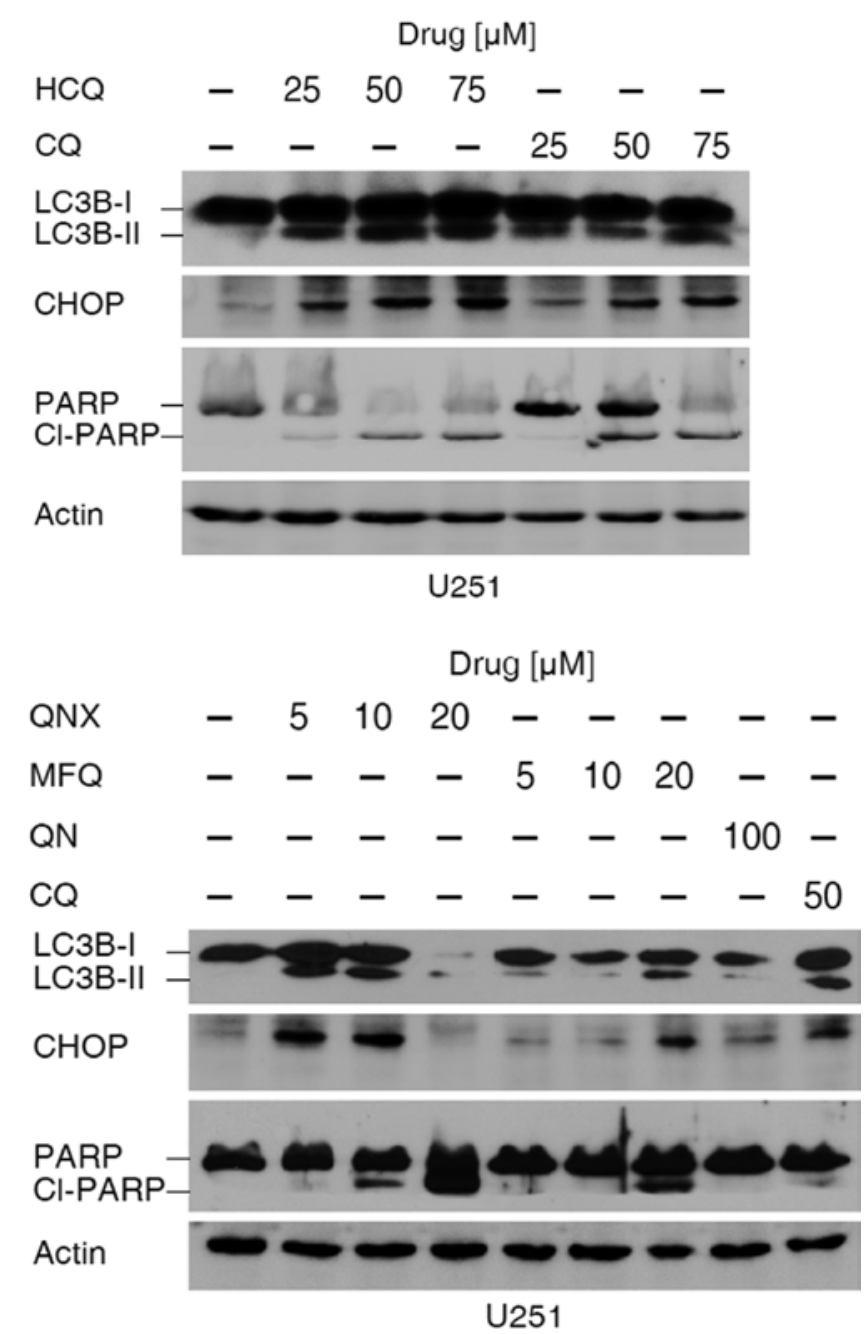

FIG. 4. Quinoline-based antimalarial compounds block autophagy and induce apoptosis. U251 glioma cells were treated with increasing concentrations of $C Q, H C Q, Q N X, M F Q$, and $Q N$ for 24 hours, and relative amounts of LC3B-I, LC3B-II, CHOP/GADD-153, and PARP were analyzed via Western blot. $\beta$-actin (actin) was used as a loading control.

$\mu \mathrm{M})$ in the presence or absence of bafilomycin A1 (200 $\mathrm{nm}$ ), a vacuolar-type proton pump inhibitor that increases the $\mathrm{pH}$ of acidic vacuoles (Fig. 5A). U251 cells treated with QNX alone resulted in an increase in intracellular punctate fluorescence. In contrast, cells treated with bafilomycin A1 exhibited no such intracellular pattern. The amount of fluorescence was quantified using flow cytometry; the results showed QNX increasing fluorescence and bafilomycin A1 decreasing the signal (Fig. 5B).

\section{In Vivo Antitumor Activity Exhibited by QBAs}

Since we demonstrated that the most potent QBA, QNX, accumulated in acidic vacuoles, we set out to determine whether this effect could be replicated in vivo. In a subcutaneous human xenograft U87 glioma model, we treated nude mice with $50 \mathrm{mg} / \mathrm{kg}$ of QNX and harvested tumors after 24 hours. Tumor cells from frozen tissue samples from mice treated with QNX exhibited fluorescence, as compared with tumor specimens from untreated animals (Fig. 6 upper). In tissues from treated animals,
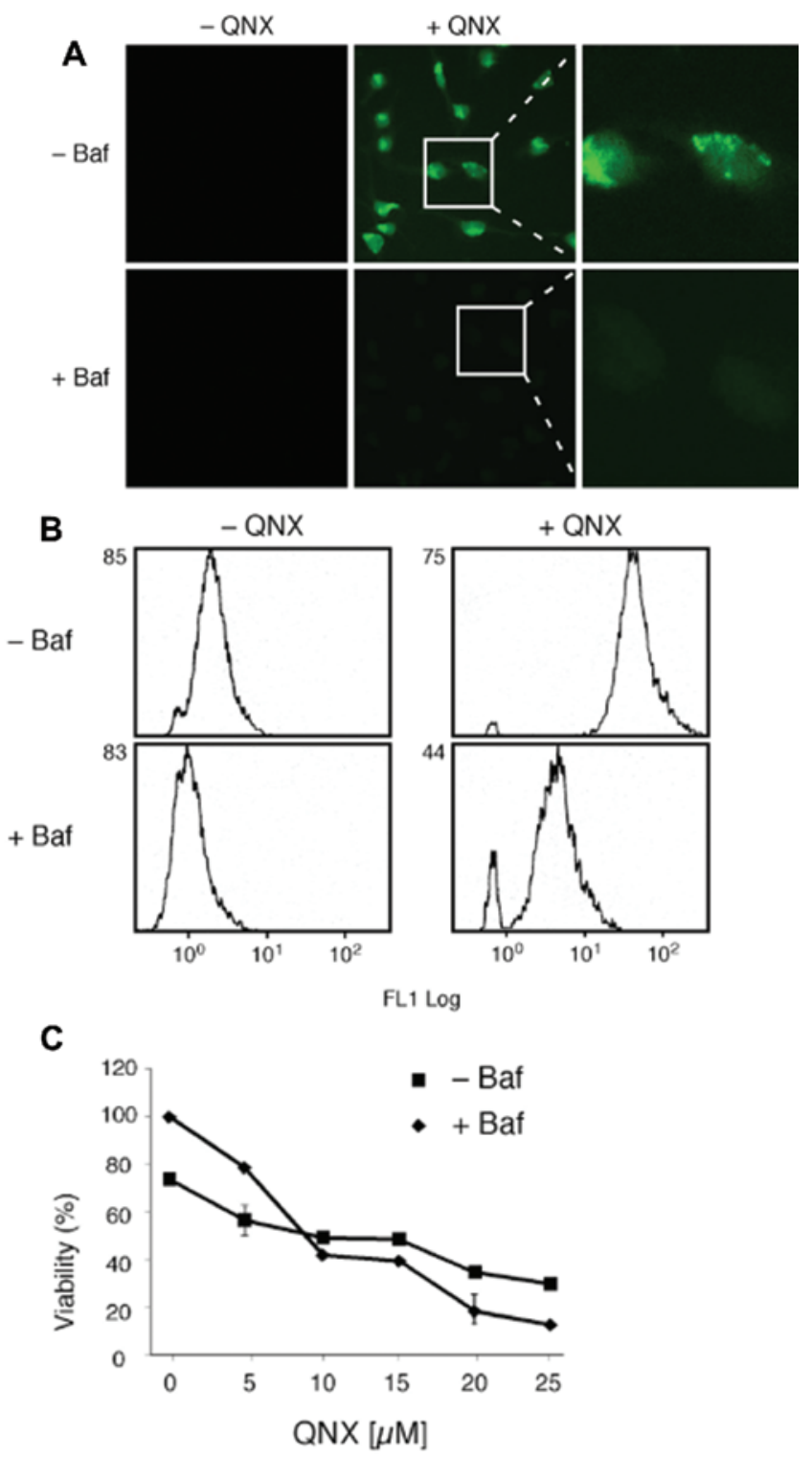

FIG. 5. Quinacrine, the most potent QBA compound, accumulates in acidic vacuoles. U251 cells were either untreated or treated with $10 \mu \mathrm{M}$ of QNX in the presence or absence of $200 \mathrm{nM}$ bafilomycin $\mathrm{A} 1$ (Baf) for 24 hours and visualized via fluorescent microscopy (A). Cellular fluorescent intensity was analyzed via flow cytometric analysis (B). Cells treated or not treated with Baf exhibited similar viability (C). - = without; $+=$ with.

the QNX formed a perinuclear punctate pattern of fluorescence, similar to the pattern observed when cells were treated with QNX in vitro. This pattern suggests that QNX selectively accumulates in tumor cell vacuoles in vivo.

To determine whether QNX can elicit the ER stress response in vivo, nude mice harboring subcutaneous U87 glioma tumors were treated with $25 \mathrm{mg} / \mathrm{kg}$ of MFQ, QNX, or QN. After 48 hours, tumors were harvested and analyzed for the ER stress marker CHOP/GADD-153 and apoptosis (Fig. 6 lower). In addition, CHOP/GADD-153 was induced in QNX-, QN-, MFQ-, and CQ-treated tumors, as compared with control tumors. The TUNEL assay for apoptosis demonstrated a significant increase in the number of 


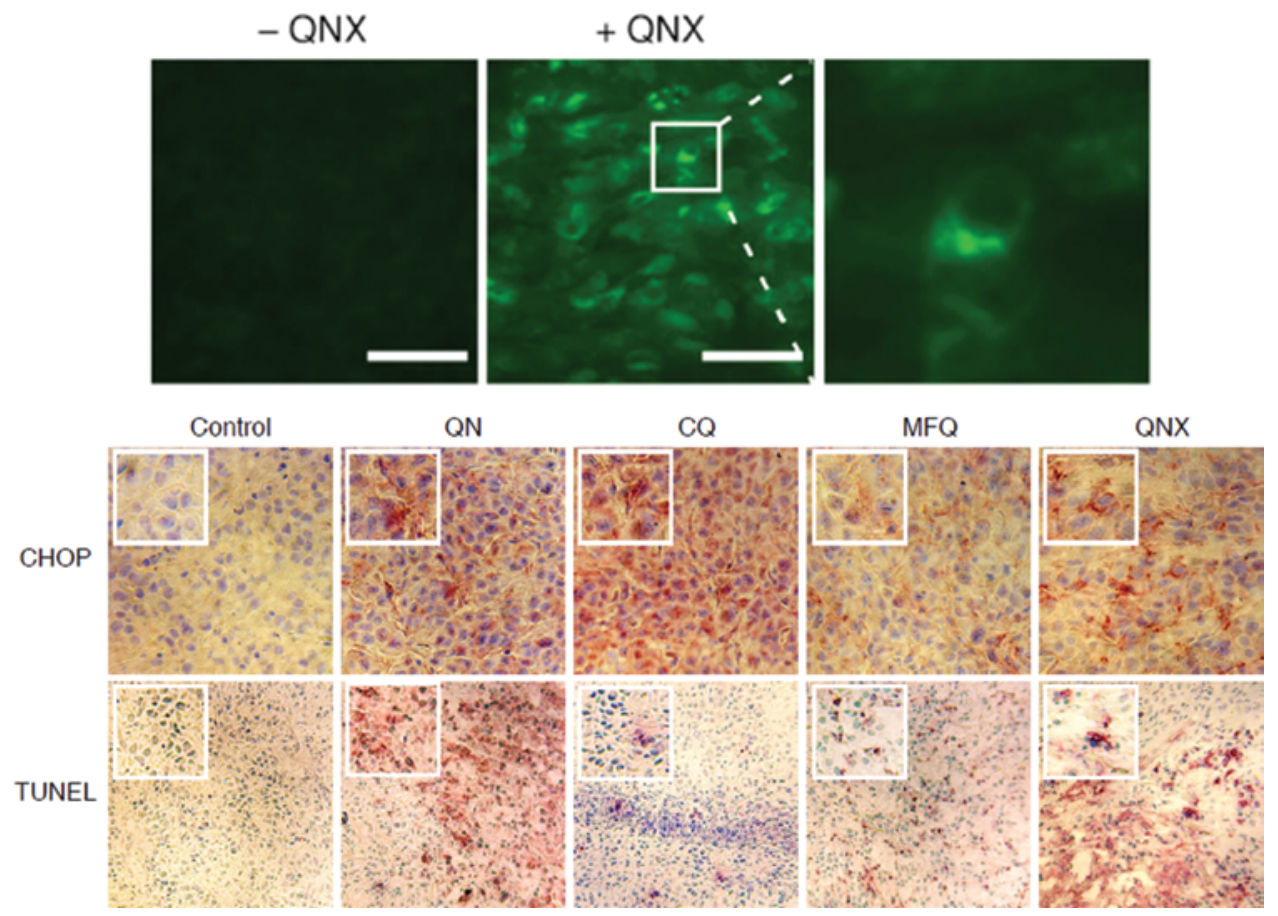

FIG. 6. Quinoline-based antimalarial compounds induce the apoptotic arm of the ER stress response in vivo. Nude mice harboring subcutaneously implanted U87 tumors were either untreated or treated with $50 \mathrm{mg} / \mathrm{kg}$ of QNX (upper). Bars $=100 \mu \mathrm{m}$. After 24 hours, the tumors were harvested, sectioned, and examined via fluorescent microscopy. In addition, nude mice harboring subcutaneously implanted U87 tumors were either untreated or treated with $25 \mathrm{mg} / \mathrm{kg} \mathrm{QN}, \mathrm{CQ}, \mathrm{MFQ}$, or QNX for 48 hours. The tumors were subsequently harvested, sectioned, and analyzed via IHC for the ER stress markers CHOP/GADD-153 or via TUNEL (an apoptosis marker; lower). The intensity of red staining in the tissue sections corresponds to the relative amount of protein present. Original magnification $\times 200$.

TUNEL-positive cells in QNX- and QN- > MFQ- > CQtreated tumors, as compared with untreated controls. These data indicate that QBAs have the ability to induce ER stress potentially leading to apoptosis in vitro and in vivo.

\section{Discussion}

The tested QBAs displayed unique cytotoxic effects in drug-resistant tumor cells. For example, all tested QBAs killed glioma cells that were highly resistant and sensitive to TMZ. These results are consistent with evidence that overexpression of multidrug-resistant pumps contributes to CQ and QN drug resistance in P. falciparum parasites. The antitumor activity of the most potent compound, QNX, correlated with accumulation in lysosomes and resultant cytotoxicity. Bafilomycin A1, a vacuolar-type ATPase proton pump inhibitor, prevented the lysosomal accumulation of QNX, thereby reducing its cytotoxic effects.

We found that all tested QBAs displayed antitumor activity similar to that shown by CQ in several tumor types. Quinacrine was the most potent compound, followed by MFQ > HCQ > CQ > QN in vitro. However, all of the tested compounds could block autophagy, as indicated by an increase in the conversion of LC3B-I to LC3B-II (a marker for autophagosome accumulation) and an increase in the levels of polyubiquitinated proteins. In addition, an increase in CHOP/GADD-153 (an ER stress proapoptotic protein) and PARP cleavage (indicator of apoptosis) revealed that apoptosis was the cause of cell death.
Autophagy is the segregation of damaged or unwanted ER and cytoplasmic constituents into autophagosomes destined for lysosomal degradation. ${ }^{30}$ This process is not only essential for eliminating intracellular pathogens via the activation of the innate and adaptive immune responses, but is also noted for its role in maintaining ER and metabolic homeostasis in tumor cells undergoing chronic hypoxia and nutrient depletion. ${ }^{31}$ Next to the proteasome, autophagy is the primary pathway for the catabolism of cellular components for amino acid and energy recycling. ${ }^{30}$ However, too much autophagy may result in cell death if the degradation of cytoplasmic content proceeds to completion..$^{12}$ Thus, the physiological relevance of autophagy in tumor cell survival and progression remains controversial. ${ }^{39}$

Although others have shown that CQ exerts its effect through proteasome inhibition, we provide evidence to the contrary (Fig. 3C). ${ }^{38}$ In this study, we showed that QBAs represent a novel class of compounds that can exert their cytotoxic effects in tumor cells through their accumulation in lysosomes, by blocking autophagosome degradation, and by causing the accumulation of cytosolic-derived protein aggregates destined for lysosomal degradation; these processes initiate ER stress-induced apoptosis. This effect is reminiscent of their ability to accumulate in the AFVs of $P$. falciparum parasites and prevent heme detoxification as a means of inciting parasite death. Thus, not only are the QBAs a novel class of autophagy inhibitors, but they also represent a class of compounds that provides a rational ap- 
proach to antitumoral chemotherapeutic regimens from a parasite-host viewpoint.

Like most parasite-host relationships, P.falciparum and tumor cells are both capable of surviving under harsh conditions within their hosts. Intraerythrocytic $P$. falciparum ingests, catabolizes, and detoxifies erythrocyte-derived hemoglobin as a means of metabolically sustaining itself. Similarly, tumor cells catabolize unwanted or unnecessary ER and cytoplasmic protein aggregates via the autophagy pathway as a means of detoxifying and metabolically sustaining itself.

Quinoline-based antimalarial compounds incite parasite death by mitigating the catabolic and detoxification systems associated with $P$. falciparum and tumor cells by accumulating in AFVs and lysosomes, respectively. Bafilomycin $\mathrm{A}$ reduces the $\mathrm{pH}$ gradient between the cytoplasm and the lysosomes of tumor cells, thereby preventing the accumulation of the QBA QNX and attenuating its cytotoxic effects. ${ }^{29} \mathrm{We}$ are currently investigating the potential adverse interactions between QBAs and proton pump inhibitors, such as omeprazole and pantoprazole, which are often prophylactically administered to prevent the onset of gastric ulcers in patients with brain tumors who are receiving dexamethasone. Whether these medications inhibit the overall efficacy of QBAs needs further assessment.

As regards malignant glioma, QBAs represent a viable therapy for newly diagnosed cases. Our demonstration that these compounds induce cytotoxicity in MGMT-negative cell lines, which subsequently become TMZ resistant, indicates that they might be used in patients in whom TMZ subsequently fails after initial responsiveness. Temozolomide induces autophagy in glioma cells as a protective mechanism; thus, inhibition of autophagy by QBAs would stimulate apoptosis. The QBA compound HCQ is currently used in clinical trials with TMZ (ClinicalTrials.gov Identifier: NCT00486603). However, our data suggest that either QNX or MFQ may be clinically superior to HCQ since both display the ability to penetrate the blood-brain barrier and possess greater cytotoxic effects than HCQ. Our data demonstrating QBAs' effectiveness in treating TMZ-resistant glioma cell lines are especially promising. Chloroquine was found to increase progression-free survival but not overall survival; all trials were performed with CQ as a single agent. The combination of quinolones with cytotoxic drugs, such as TMZ, has not been widely used. A possible advantage of the QBA therapy is that the systemic side effects of QBAs are fairly minor. We selected cell lines that were initially MGMT negative as they represented lines that would have the best response to the current standard of care. ${ }^{23}$ However, when the cell lines became TMZ resistant, we demonstrated that QBAs maintain activity. These data suggest that patients whose disease progresses on standard TMZ chemotherapy may use QBAs either in combination with TMZ or as stand-alone therapy. Moreover, QBAs' effectiveness in treating glioma cells regardless of their p53 status suggests its effectiveness in primary and secondary glioblastomas.

Finally, multilevel manipulation of ER stress and autophagy with combination treatments may severely enhance ER stress and greatly increase tumor cell death. Others have shown that MFQ inhibits sarcoplasmic/ER calcium ATPase (SERCA), thereby causing calcium release from the ER, an increase in misfolded proteins, and induction of the ER stress response. ${ }^{10,16}$ Thus, MFQ's multilevel effect of increasing the burden of unfolded ER proteins targeted for lysosomal degradation while blocking autophagy and aggravating ER stress may contribute to greater potency, in comparison with the other QBAs. ${ }^{35} \mathrm{We}$ previously demonstrated that combinations of chemotherapeutic compounds that manipulate the ER stress response at multiple levels hold clinical promise by synergistically inciting cell death. ${ }^{13,24,34}$ Now that we have established a novel class of autophagy inhibitors, it will be a matter of further characterizing the unique secondary qualities of each compound as a strategy for optimizing the activation of ER stress in combination with other clinically useful chemotherapies, such as proteasome and SERCA inhibitors, as a means of reducing the tumor burden while minimizing systemic side effects.

\section{Conclusions}

In summary, the antimalarial compounds investigated in this study hold promise as a novel class of autophagy inhibitors for the treatment of newly diagnosed TMZ-sensitive and recurrent TMZ-resistant gliomas.

\section{References}

1. Amaravadi RK, Yu D, Lum JJ, Bui T, Christophorou MA, Evan GI, et al: Autophagy inhibition enhances therapyinduced apoptosis in a Myc-induced model of lymphoma. $\mathbf{J}$ Clin Invest 117:326-336, 2007

2. Bachhawat K, Thomas CJ, Surolia N, Surolia A: Interaction of chloroquine and its analogues with heme: an isothermal titration calorimetric study. Biochem Biophys Res Commun 276:1075-1079, 2000

3. Baird JK: Effectiveness of antimalarial drugs. N Engl J Med 352:1565-1577, 2005

4. Baranova AM: [Current antimalarial drugs recommended by the World Health Organization for the treatment and prevention of malaria.] Med Parazitol (Mosk) 3:56-59, 2007 (Russian)

5. Barraud de Lagerie S, Comets E, Gautrand C, Fernandez C, Auchere D, Singlas E, et al: Cerebral uptake of mefloquine enantiomers with and without the P-gp inhibitor elacridar (GF1210918) in mice. Br J Pharmacol 141:1214-1222, 2004

6. Briceño E, Calderon A, Sotelo J: Institutional experience with chloroquine as an adjuvant to the therapy for glioblastoma multiforme. Surg Neurol 67:388-391, 2007

7. Brocks DR, Mehvar R: Stereoselectivity in the pharmacodynamics and pharmacokinetics of the chiral antimalarial drugs. Clin Pharmacokinet 42:1359-1382, 2003

8. Bursch W, Karwan A, Mayer M, Dornetshuber J, Fröhwein U, Schulte-Hermann R, et al: Cell death and autophagy: cytokines, drugs, and nutritional factors. Toxicology 254:147157, 2008

9. Carew JS, Nawrocki ST, Cleveland JL: Modulating autophagy for therapeutic benefit. Autophagy 3:464-467, 2007

10. Caridha D, Yourick D, Cabezas M, Wolf L, Hudson TH, Dow GS: Mefloquine-induced disruption of calcium homeostasis in mammalian cells is similar to that induced by ionomycin. Antimicrob Agents Chemother 52:684-693, 2008

11. Chauhan PM, Srivastava SK: Present trends and future strategy in chemotherapy of malaria. Curr Med Chem 8:15351542,2001

12. Chen N, Karantza-Wadsworth V: Role and regulation of au- 
tophagy in cancer. Biochim Biophys Acta 1793:1516-1523, 2009

13. Cho HY, Thomas S, Golden EB, Gaffney KJ, Hofman FM, Chen TC, et al: Enhanced killing of chemo-resistant breast cancer cells via controlled aggravation of ER stress. Cancer Lett 282:87-97, 2009

14. Cho HY, Wang W, Jhaveri N, Lee DJ, Sharma N, Dubeau L, et al: NEO212, temozolomide conjugated to perillyl alcohol, is a novel drug for effective treatment of a broad range of temozolomide-resistant gliomas. Mol Cancer Ther 13: 2004-2017, 2014

15. Degtyarev M, De Mazière A, Orr C, Lin J, Lee BB, Tien JY, et al: Akt inhibition promotes autophagy and sensitizes PTEN-null tumors to lysosomotropic agents. J Cell Biol 183:101-116, 2008

16. Dow GS, Caridha D, Goldberg M, Wolf L, Koenig ML, Yourick DL, et al: Transcriptional profiling of mefloquine-induced disruption of calcium homeostasis in neurons in vitro. Genomics 86:539-550, 2005

17. Easterbrook M: Detection and prevention of maculopathy associated with antimalarial agents. Int Ophthalmol Clin 39:49-57, 1999

18. Elliott DA, McIntosh MT, Hosgood HD III, Chen S, Zhang G, Baevova P, et al: Four distinct pathways of hemoglobin uptake in the malaria parasite Plasmodium falciparum. Proc Natl Acad Sci U S A 105:2463-2468, 2008

19. Fitch CD, Russell NV: Accelerated denaturation of hemoglobin and the antimalarial action of chloroquine. Antimicrob Agents Chemother 50:2415-2419, 2006

20. Frosch T, Schmitt M, Bringmann G, Kiefer W, Popp J: Structural analysis of the anti-malaria active agent chloroquine under physiological conditions. J Phys Chem B 111:1815-1822, 2007

21. Gayrard V, Picard-Hagen N, Viguié C, Laroute V, Andréoletti O, Toutain PL: A possible pharmacological explanation for quinacrine failure to treat prion diseases: pharmacokinetic investigations in a ovine model of scrapie. Br J Pharmacol 144:386-393, 2005

22. Golenser J, McQuillan J, Hee L, Mitchell AJ, Hunt NH: Conventional and experimental treatment of cerebral malaria. Int J Parasitol 36:583-593, 2006

23. Hegi ME, Liu L, Herman JG, Stupp R, Wick W, Weller M, et al: Correlation of O6-methylguanine methyltransferase (MGMT) promoter methylation with clinical outcomes in glioblastoma and clinical strategies to modulate MGMT activity. J Clin Oncol 26:4189-4199, 2008

24. Kardosh A, Golden EB, Pyrko P, Uddin J, Hofman FM, Chen TC, et al: Aggravated endoplasmic reticulum stress as a basis for enhanced glioblastoma cell killing by bortezomib in combination with celecoxib or its non-coxib analogue, 2,5-dimethyl-celecoxib. Cancer Res 68:843-851, 2008

25. Kardosh A, Soriano N, Liu YT, Uddin J, Petasis NA, Hofman FM, et al: Multitarget inhibition of drug-resistant multiple myeloma cell lines by dimethyl-celecoxib (DMC), a nonCOX-2 inhibitory analog of celecoxib. Blood 106:4330 4338,2005

26. Lazarus MD, Schneider TG, Taraschi TF: A new model for hemoglobin ingestion and transport by the human malaria parasite Plasmodium falciparum. J Cell Sci 121:1937-1949, 2008

27. Lim HS, Im JS, Cho JY, Bae KS, Klein TA, Yeom JS, et al: Pharmacokinetics of hydroxychloroquine and its clinical implications in chemoprophylaxis against malaria caused by Plasmodium vivax. Antimicrob Agents Chemother 53: 1468-1475, 2009

28. Maguire JD, Krisin, Marwoto H, Richie TL, Fryauff DJ, Baird JK: Mefloquine is highly efficacious against chloroquine-resistant Plasmodium vivax malaria and Plasmodium falciparum malaria in Papua, Indonesia. Clin Infect Dis 42:1067-1072, 2006
29. Marceau F, Bawolak MT, Bouthillier J, Morissette G: Vacuolar ATPase-mediated cellular concentration and retention of quinacrine: a model for the distribution of lipophilic cationic drugs to autophagic vacuoles. Drug Metab Dispos 37:22712274, 2009

30. Münz C: Enhancing immunity through autophagy. Annu Rev Immunol 27:423-449, 2009

31. Orvedahl A, Levine B: Eating the enemy within: autophagy in infectious diseases. Cell Death Differ 16:57-69, 2009

32. Pham YT, Nosten F, Farinotti R, White NJ, Gimenez F: Cerebral uptake of mefloquine enantiomers in fatal cerebral malaria. Int J Clin Pharmacol Ther 37:58-61, 1999

33. Sanchez CP, McLean JE, Stein W, Lanzer M: Evidence for a substrate specific and inhibitable drug efflux system in chloroquine resistant Plasmodium falciparum strains. Biochemistry 43:16365-16373, 2004

34. Schönthal AH: Endoplasmic reticulum stress and autophagy as targets for cancer therapy. Cancer Lett 275:163-169, 2009

35. Sharma N, Thomas S, Golden EB, Hofman FM, Chen TC, Petasis NA, et al: Inhibition of autophagy and induction of breast cancer cell death by mefloquine, an antimalarial agent. Cancer Lett 326: 143-154, 2012

36. Shono T, Tofilon PJ, Schaefer TS, Parikh D, Liu TJ, Lang FF: Apoptosis induced by adenovirus-mediated p53 gene transfer in human glioma correlates with site-specific phosphorylation. Cancer Res 62:1069-1076, 2002

37. Slater AF: Chloroquine: mechanism of drug action and resistance in Plasmodium falciparum. Pharmacol Ther 57:203235,1993

38. Sprangers R, Li X, Mao X, Rubinstein JL, Schimmer AD, Kay LE: TROSY-based NMR evidence for a novel class of 20 S proteasome inhibitors. Biochemistry 47:6727-6734, 2008

39. Tsuchihara K, Fujii S, Esumi H: Autophagy and cancer: dynamism of the metabolism of tumor cells and tissues. Cancer Lett 278:130-138, 2009

40. Warhurst DC, Craig JC, Adagu IS, Meyer DJ, Lee SY: The relationship of physico-chemical properties and structure to the differential antiplasmodial activity of the cinchona alkaloids. Malar J 2:26, 2003

41. Webster RV, Craig JC, Shyamala V, Kirby GC, Warhurst DC: Antimalarial activity of optical isomers of quinacrine dihydrochloride against chloroquine-sensitive and -resistant Plasmodium falciparum in vitro. Biochem Pharmacol 42 Suppl:S225-S227, 1991

42. Zagzag D, Nomura M, Friedlander DR, Blanco CY, Gagner JP, Nomura N, et al: Geldanamycin inhibits migration of glioma cells in vitro: a potential role for hypoxia-inducible factor (HIF-1alpha) in glioma cell invasion. J Cell Physiol 196:394-402, 2003

\section{Author Contributions}

Conception and design: Chen, Golden, Louie, Schönthal. Acquisition of data: Golden, Cho, Louie. Analysis and interpretation of data: all authors. Drafting the article: Golden, Schönthal. Critically revising the article: Chen, Cho, Hofman, Schönthal. Reviewed submitted version of manuscript: Chen, Cho, Hofman, Louie. Approved the final version of the manuscript on behalf of all authors: Chen. Statistical analysis: Louie. Administrative/ technical/material support: Chen, Cho, Hofman, Schönthal. Study supervision: Chen, Hofman, Schönthal.

\section{Correspondence}

Thomas C. Chen, University of Southern California, 1200 N. State St. \#5046, Los Angeles, CA 90033. email: tchen68670@ gmail.com. 